\title{
Development of digastric muscles in human foetuses: a review and findings in the flexor digitorum superficialis muscle
}

\author{
J.F. Rodríguez-Vázquez , Z.W. Jin², P. Zhao², G. Murakami1, 3, X.W. Li , Y. Jin \\ ${ }^{1}$ Department of Anatomy and Human Embryology, Institute of Embryology, Faculty of Medicine, \\ Complutense University, Madrid, Spain \\ ${ }^{2}$ Department of Anatomy, Wuxi School of Medicine, Jiangnan University, Wuxi, China \\ ${ }^{3}$ Division of Internal Medicine, Iwamizawa Asuka Hospital, Iwamizawa, Japan \\ ${ }^{4}$ Department of Anatomy, Histology and Embryology, Yanbian University Medical College, Yanji, China
}

[Received: 13 June 2017; Accepted: 2 August 2017]

The digastricus and omohyoideus muscles are digastric muscles with two muscle bellies. An insertion tendon of the posterior belly becomes an intermediate tendon in digastricus muscles, whereas a single band-like muscle in omohyoideus muscles may later be interrupted by an intermediate tendon, possibly due to muscle cell death caused by mechanical stress. In human foetuses, an intermediate tendon provides the temporal origins of the tensor veli palatini and tensor tympani muscles. Some reptiles, including snakes, carry multiple series of digastric-like axial muscles, in which each intersegmental septum is likely to become an intermediate tendon. These findings indicate that many pathways are involved in the development of digastric muscles. A review of these morphologies suggested that the flexor digitorum superficialis (FDS) muscle was a digastric muscle, although the intermediate tendon may not be visible in the surface view in adults. The present observations support the hypothesis that the proximal anlage at the elbow develops into a deep muscle slip to a limited finger, while the distal anlage at the wrist develops into the other slips. The findings suggest that, in the FDS muscle, the proximal and distal bellies of the embryonic digastric muscle fuse together to form a laminar structure, in which muscle slips accumulate from the palmar to the deep side of the forearm. (Folia Morphol 2018; 77, 2: 362-370)

Key words: intermediate tendon, digastricus muscle, omohyoideus muscle, tensor veli palatini muscle, tensor tympani muscle, flexor digitorum superficialis muscle, human foetus

\section{INTRODUCTION}

The digastricus and omohyoideus muscles at the head and neck are both digastric muscles, each with two muscle bellies, but their foetal development differs markedly. We previously reported that an insertion tendon of the posterior belly innervated by the facial nerve becomes the intermediate tendon of the digastricus muscle, as the hypoglossal nerve and stylohyoid muscle make it difficult for the posterior belly to obtain the hyoid attachment [10]. Simultaneously, muscle fibres of the anterior belly supplied by the trigeminal nerve attach to and finally cover the tendon of posterior belly (Fig. 1A). Thus, in midterm foetuses, various numbers of muscle fibres of the anterior belly are maintained in and along the future intermediate tendon. In contrast, the omohyoideus 


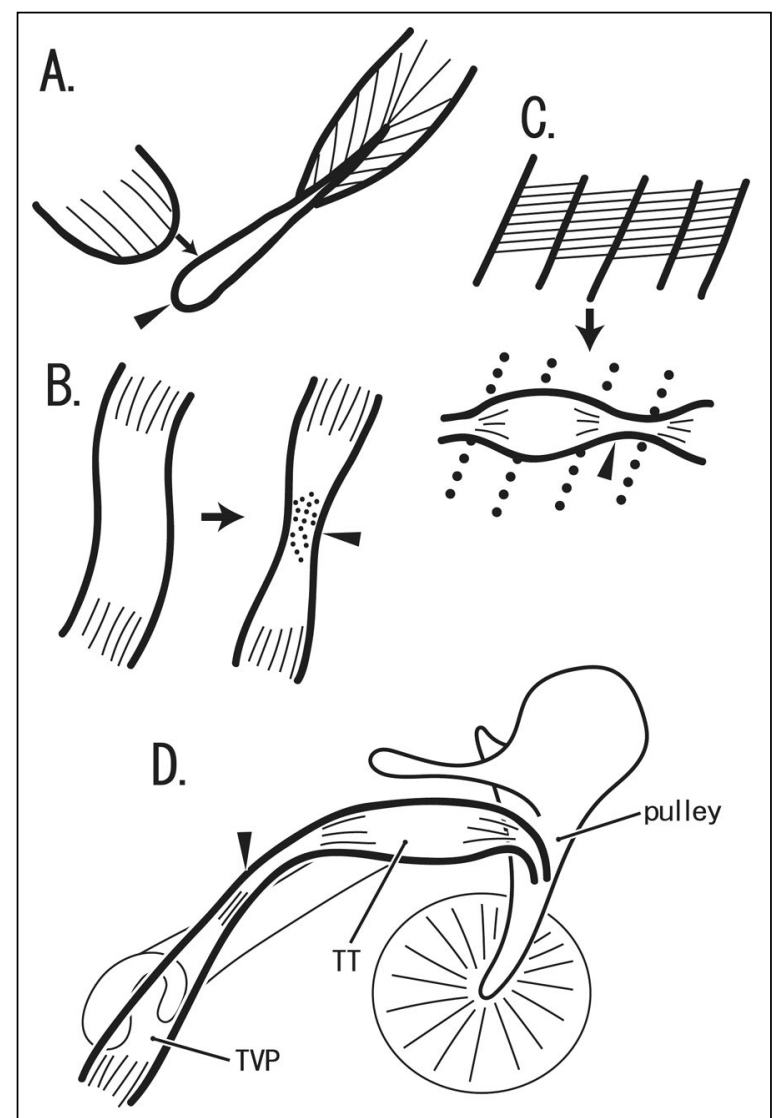

Figure 1. Reported digastric muscles including axial muscles in reptiles and their hypothetical development; $\mathbf{A}$. The digastricus muscle in the head and neck. The later-developing anterior belly attaches to a floating end (arrowhead) of the long tendon of the posterior belly; B. The omohyoid muscle in the neck. The intermediate tendon (arrowhead) appears later in the midcourse of a belt-like muscle. C. In some reptiles, segmental axial muscles seem to change to multiple muscle bellies connected with an intermediate tendon (arrowhead); $\mathbf{D}$. The tensor veli palatini (TVP) and tensor tympani (TT) muscles. The intermediate tendon (arrowhead) is evident at embryonic stage. The tendon inserted into the malleus changed direction at a pulley.

was found to be a belt-like muscle in midterm foetuses, but the intermediate tendon develops later at an angulation site, at which the muscle belt attaches to the carotid sheath (Fig. 1B) [10]. Thus mechanical stress may induce fibrosis, altering some of the muscle fibres within the tendon. The different branches of the ansa cervicalis nerve supply the upper and lower parts of the initial belt-like muscle, respectively, and the upper and lower groups of muscle fibres are interdigitated close to the centre of the future intermediate tendon. This configuration, with interrupted muscle fibres, is termed an "in-series" or "non-spanning" muscle, whereas a muscle in which all muscle fibres

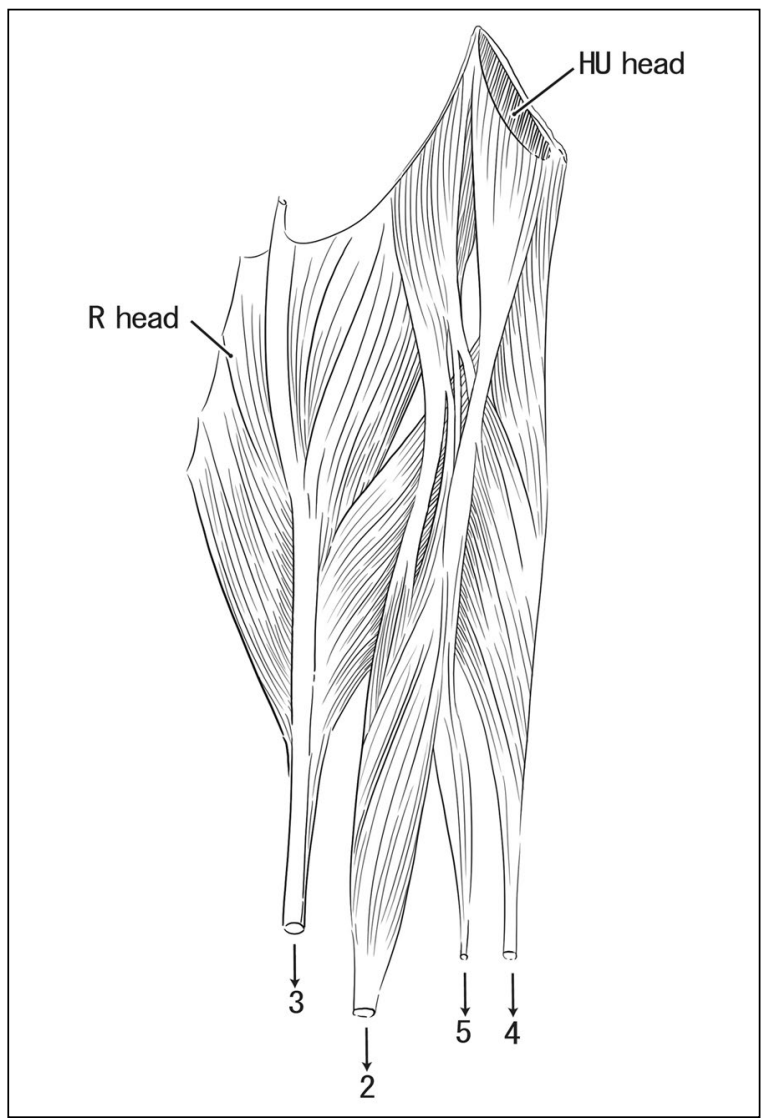

Figure 2. Schematic representation of the flexor digitorum superficialis muscle. The muscle was viewed from its posterior or deep aspect after slight dissection. The top of the figure corresponds to the proximal side of the forearm. The labels 2, 3, 4 and 5 indicate the finger for insertion. The proximal part of the muscle slip to the second finger and two intermediate or thick intramuscular tendons are shown. HU head — humero-ulnar head; R head — radial head. Modified from Frohse and Fränkel [4].

extend the entire length of the muscle is termed a "spanning" muscle [7, 19].

Our group recently demonstrated that the tensor veli palatini and tensor tympani muscles in human foetuses originate from an intermediate tendon (Fig. 1D) [15]. However, these intermediate tendons are not likely to be maintained in adults [1]. In addition, some reptiles, including snakes, carry multiple series of digastric-like axial muscles, in which each intersegmental septum is likely to develop into an intermediate tendon (Fig. 1C). The axial musculature of reptiles, especially muscles corresponding to the intercostalis externi, levator costae and iliocostalis in mammals, was found to contain well-developed intersegmental septa, with each myotome supplied by two adjacent spinal nerves $[5,13]$. Overall, these find- 
ings indicate that digastric muscles and intermediate tendons develop via multiple pathways. The first aim of this study was to review the development of the digastric muscle and intermediate tendon.

The flexor digitorum superficialis (FDS) muscle in adults is composed of several muscle slips that accumulate in a 3-dimensional manner. Figure 2 shows these slips after slight dissection for 2-dimensional extension. The muscle slip arrangement of FDS muscles varies widely and can include absence of a tendon to the fifth finger $[4,14,20]$. The basic double-layered configuration consists of a deep layer containing muscle slips for the second and third fingers and regarded as a digastric configuration, and a superficial layer comprised of slips for the third and fourth fingers. Although the intermediate tendon cannot be visualised by the surface view, the masked intermediate tendon always connects a deep muscle slip for the second finger to the distal parts of the FDS muscle [14, 20]. These findings suggested that a nerve branch to the second finger muscle slip (ramus superior [4]) originates from the median nerve at a site distant from the others (rami medius and inferior) and that the former branch is likely bundled with a nerve to the palmaris longus muscle.

The results of two embryologic studies suggested that the FDS muscle was arranged in a digastric configuration $[3,6]$, in that the proximal anlage at the elbow developed into a posterior muscle slip, while the distal anlage at the wrist developed into the other slips. Other studies, however, indicated the FDS originated from a single anlage in the deep layer of the forearm [11, 12]. The digastric morphology of the FDS muscle is therefore likely to be evident in the embryonic stage. Thus, the second aim of this study was to reexamine the human FDS using serial histological sections of embryos and foetuses.

\section{MATERIALS AND METHODS}

This study was performed in accordance with the provisions of the Declaration of Helsinki 1995 (as revised in 2013). Serial sections of the upper extremities were obtained from 30 embryos and foetuses of gestational age 6-8 weeks (crown rump length [CRL] 15-35 mm). Twenty of these 30 specimens were of Spanish origin, with the other 10 obtained in China. The Spanish specimens were part of the large collection kept at the Embryology Institute of the Universidad Complutense, Madrid, and were the products of miscarriages and ectopic pregnancies in the Department of Obstetrics at the University.
The upper extremities of the 20 Spanish specimens had been cut serially; 15 longitudinally-sectioned and 5 transversely-sectioned specimens of the forearm and hand were chosen. In the former, however, the sectional planes varied (sagittal, frontal or oblique) depending on the posture of the upper extremity. Most of these sections were stained with haematoxylin and eosin (HE), with other stained with azan, orange $\mathrm{G}$ or silver stain. The study protocol was approved by the ethics committee of Complutense University (B08/374).

The remaining 10 foetuses, all of gestational age 8 weeks were donated by their families to the Department of Anatomy, Yanbian University Medical College, Yanji, China, until 2016. The use of these samples for research was approved by the ethics committee of Yanbian University (No. BS-13-35). These foetuses were obtained by induced abortion, after which the mother was orally informed by an obstetrician at the college teaching hospital of the possibility of donating the foetus for research; no attempt was made to actively encourage the donation. After the mother agreed, the foetus was assigned a specimen number and stored in $10 \% \mathrm{w} / \mathrm{w}$ neutral formalin solution for more than 1 month. Because of specimen number randomisation, there was no possibility of contacting the family at a later date. After dividing the body into parts, the forearm and hand were sectioned transversely at 20 micron intervals ( 5 microns in thickness) and stained with HE. Sections were observed and photographed with a Nikon Eclipse 80.

\section{RESULTS}

\section{Observations of longitudinal sections}

Forearm length was very short in foetuses of gestational age 6-8 weeks, being about half the longitudinal length of the hand and finger (Figs. 2-4). The distal anlage of the FDS muscle could be clearly identified as a large spindle-shaped mass at the level of the carpal bones (Figs. 3E, F; 4C, D). According to tendon directions of the flexor digitorum profundus (FDP) muscle, the distal anlage of the FDS muscle extended longitudinally on the superficial side of the deep tendons to the fourth and fifth fingers (Figs. 3E; 4C). Thus, the distal anlage was located almost in the ulnar half of the hand and wrist. After providing branches to the FDS muscle, the median nerve appeared to run distally on the superficial side of the anlage (Figs. 3D; 4A). The distal nerve was therefore regarded as a digital cutaneous nerve. 

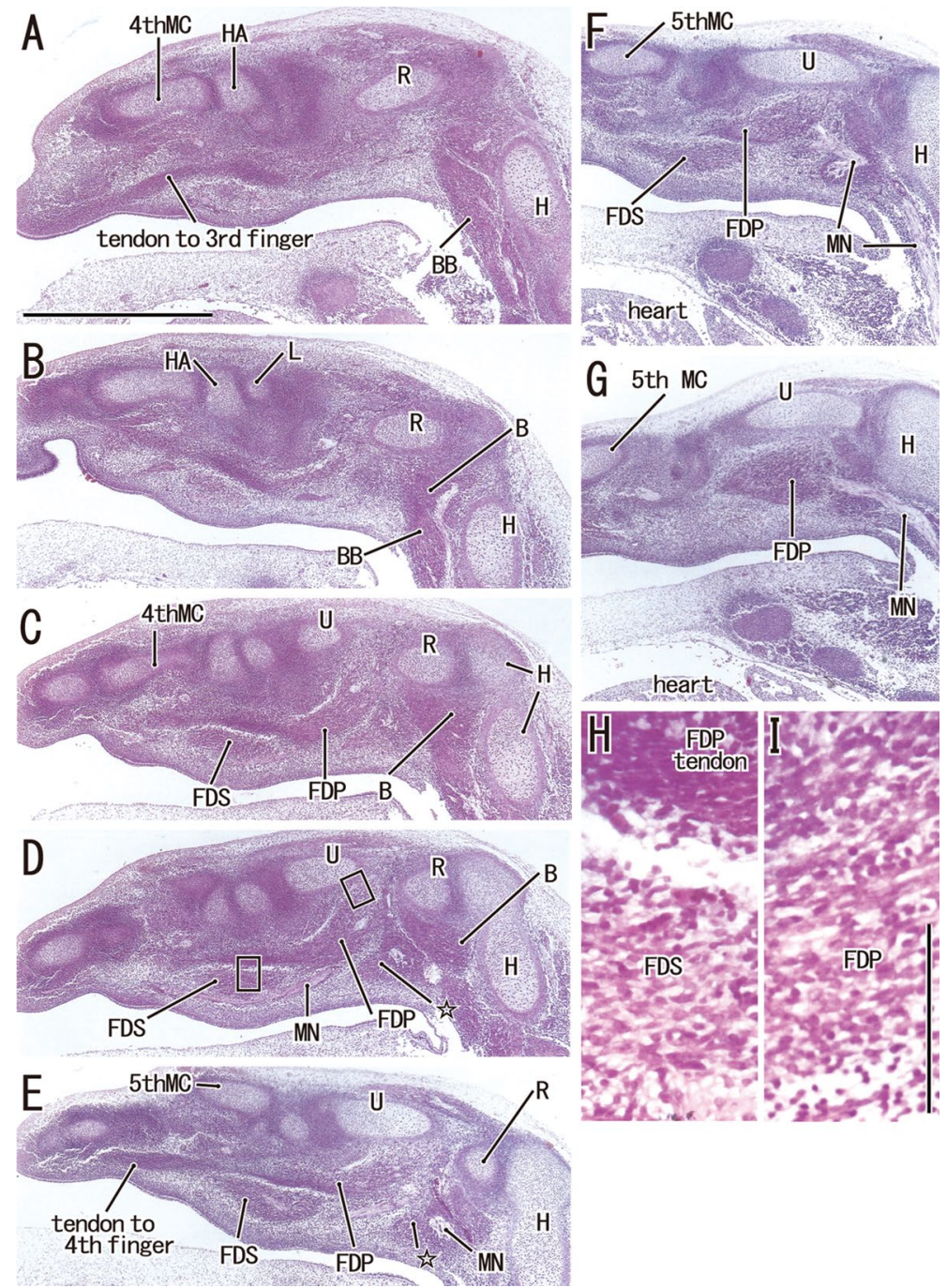

Figure 3. A-G. Muscle anlagen in the forearm and hand: almost sagittal sections from a specimen of crown rump length $15 \mathrm{~mm}$ (gestational age 6 weeks). A, G. The most radial and ulnar sides of the figure, respectively. Panels $A-G$ were prepared at the same magnification (scale bar in panel $A, 1 \mathrm{~mm}$ ). Intervals between panels were $0.1 \mathrm{~mm}$. Panels $\mathbf{H}$ and $I$ are higher magnification views of the squares in panel $D$ (scale bar in panel I, $0.1 \mathrm{~mm}$ ). A distal anlage of the flexor digitorum superficialis (FDS) muscle is clearly seen in panels $C-F$, whereas a small anlage (asterisk in panels $\mathrm{D}$ and $\mathrm{E}$ ) near the median nerve (MN) at the elbow appears to correspond to the proximal anlage. The FDS anlage does not accompany tendons. Two long tendons of the flexor digitorum profundus (FDP) muscle, to the third and fourth fingers, are present. In these flexors, primary myotubes appear to be differentiated (panels $\mathrm{H}$ and I). Panel $\mathrm{D}$ shows that the median nerve apparently gives rise to a thick cutaneous branch, whereas panel $F$ shows that the flexor digitorum profundus muscle is attached to the muscle in the humero-ulnar joint; $\mathrm{B}$ - brachialis muscle; $\mathrm{BB}$ - biceps brachii muscle; $\mathrm{H}$ - humerus; $\mathrm{HA}$ - hamate; L - lunate; $\mathrm{MC}$ - metacarpal bone; U — ulna.
In contrast to the distal anlage, the proximal anlage of the FDS was difficult to identify because, in strict sagittal sections, not only the pronator teres and flexor carpi radialis muscles but the thick median nerve were packed together in a narrow space near the medial epicondyle of the humerus (Figs. 3D-F). After identifying the pronator teres as a wide muscle plate extending between the humerus and radius, depending on suitable flexion and rotation at the elbow (Figs. 4F; 5A), the proximal anlage of the FDS could be distinguished from the FDP anlage on the superficial side of the median nerve (Fig. 5B, C). The distal and proximal anlagen of the FDS muscle were connected by a weakly eosinophilic, slender tissue on the superficial side of the FDP and constituting a candidate intermediate tendon of the FDS muscle (Figs. 3, 4; 5A). Parts of the FDP supplied by the ulnar nerve (Fig. 5F, G) were distant from and on the ulnar side of the proximal anlage of the FDS. The humeral head of the pronator teres was large but its ulnar head appeared to be absent from all specimens. Similarly, we were unable to demonstrate the palmaris longus muscle in longitudinal sections. The distal and proximal anlagen of the FDS as well as the anlage of the FDP contained primary myotubes.

\section{Observations in transverse sections}

All antebrachial muscles, including the palmaris longus and pronator quadratus muscles were visible on transverse sections. A tendinous plate or a tendon common to the FDP and flexor pollicis longus was evident at the wrist (Figs. 6, 7). Figure 6 shows the early stage morphology of intermediate tendons of the FDS, consisting of a distal mass of primary myotubes interrupted by a candidate intermediate tendon (Fig. 6D), with the latter extending to the proximal 

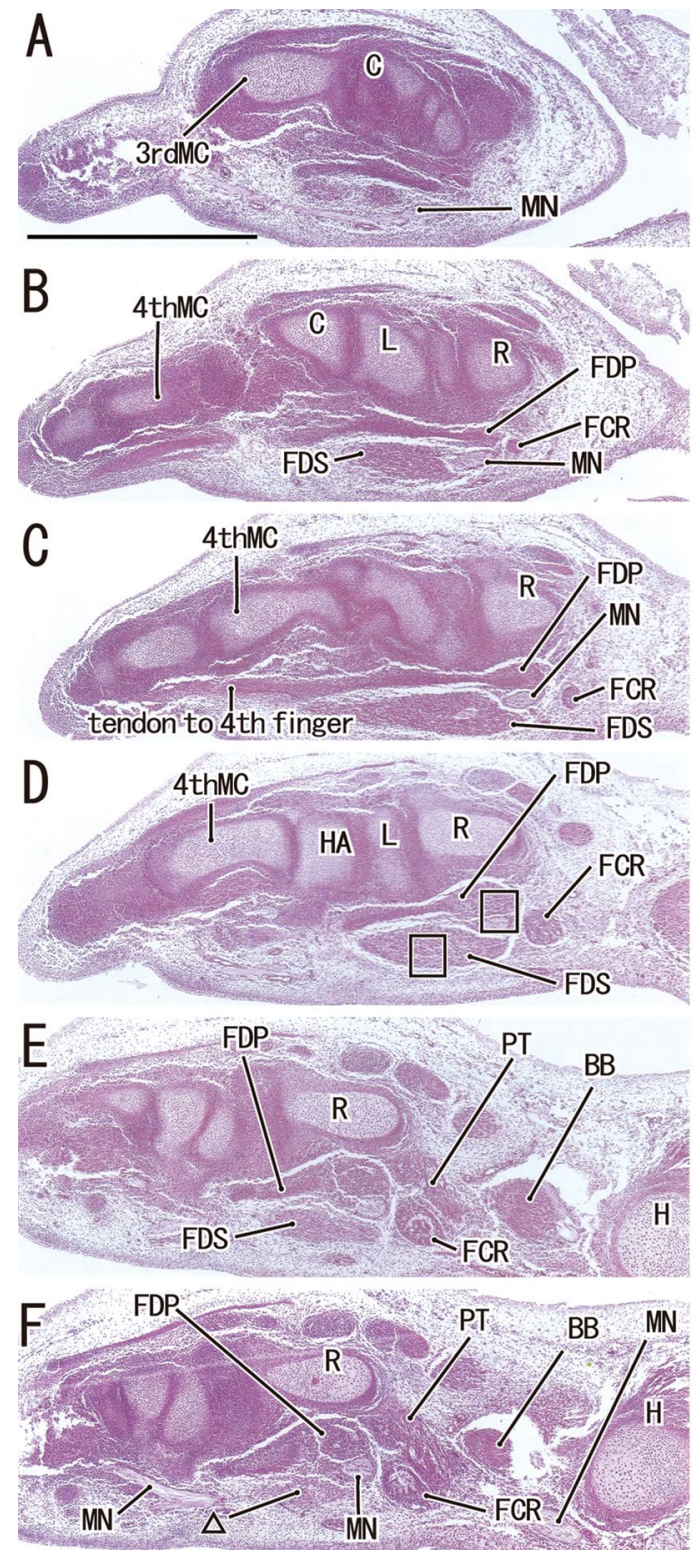

mass of myotubes adjacent to the flexor carpi radialis. The FDP on the proximal side of the candidate intermediate tendon contained multiple intramuscular tendonlike structures (Figs. 6B-D; 7H-K), with parts extending into the FDS. This prevented the identification of the intermediate tendon of the FDS in many samples. Digital tendons of the FDP were well differentiated at the level of the metacarpal bone, whereas FDS tendons were weakly eosinophilic and poorly differentiated (Fig. 7B-D). Proximally, the initial FDS tendons joined to provide 2-4 muscle fascicles (Figs. 7F, G).

Near the medial epicondyle of the humerus, several muscles, including the flexor carpi radialis, palmaris longus, pronator teres, FDS and flexor carpi
Figure 4. A-H. Muscle anlagen in the forearm and hand: almost sagittal sections from a specimen of crown rump length $25 \mathrm{~mm}$ (gestational age 7 weeks). A, F. The most radial and ulnar sides of the figure, respectively. Panels A-F were prepared at the same magnification (scale bar in panel $A$, $1 \mathrm{~mm}$ ). Intervals between panels are $0.2 \mathrm{~mm}$ (A-B, B-C, C-D, D-E) and $0.1 \mathrm{~mm}(\mathrm{E}-\mathrm{F})$. Panels $G$ and $H$ are higher magnification views of the squares in panel $D$ (scale bar in panel G, $0.1 \mathrm{~mm}$ ). A distal anlage of the flexor digitorum superficialis muscle (FDS) is clearly seen in panels $B-D$, but is weakly stained in panel $E$ and became tendinous in panel $\mathrm{F}$ (triangle representing a candidate intermediate tendon). The median nerve (MN) enters a space between the superficial and deep flexors (panels $C$ and $D$ ). In flexors, the primary myotubes appear to be differentiated (panels $\mathrm{G}$ and $\mathrm{H}$ ); $\mathrm{BB}$ - biceps brachii muscle; $\mathrm{C}$ - capitate; FCR — flexor carpi radialis muscle; FDP flexor digitorum profundus muscle; $\mathrm{H}-$ humerus; HA — hamate; L — lunate; PT pronator teres muscle; $\mathrm{R}$ - radius. 

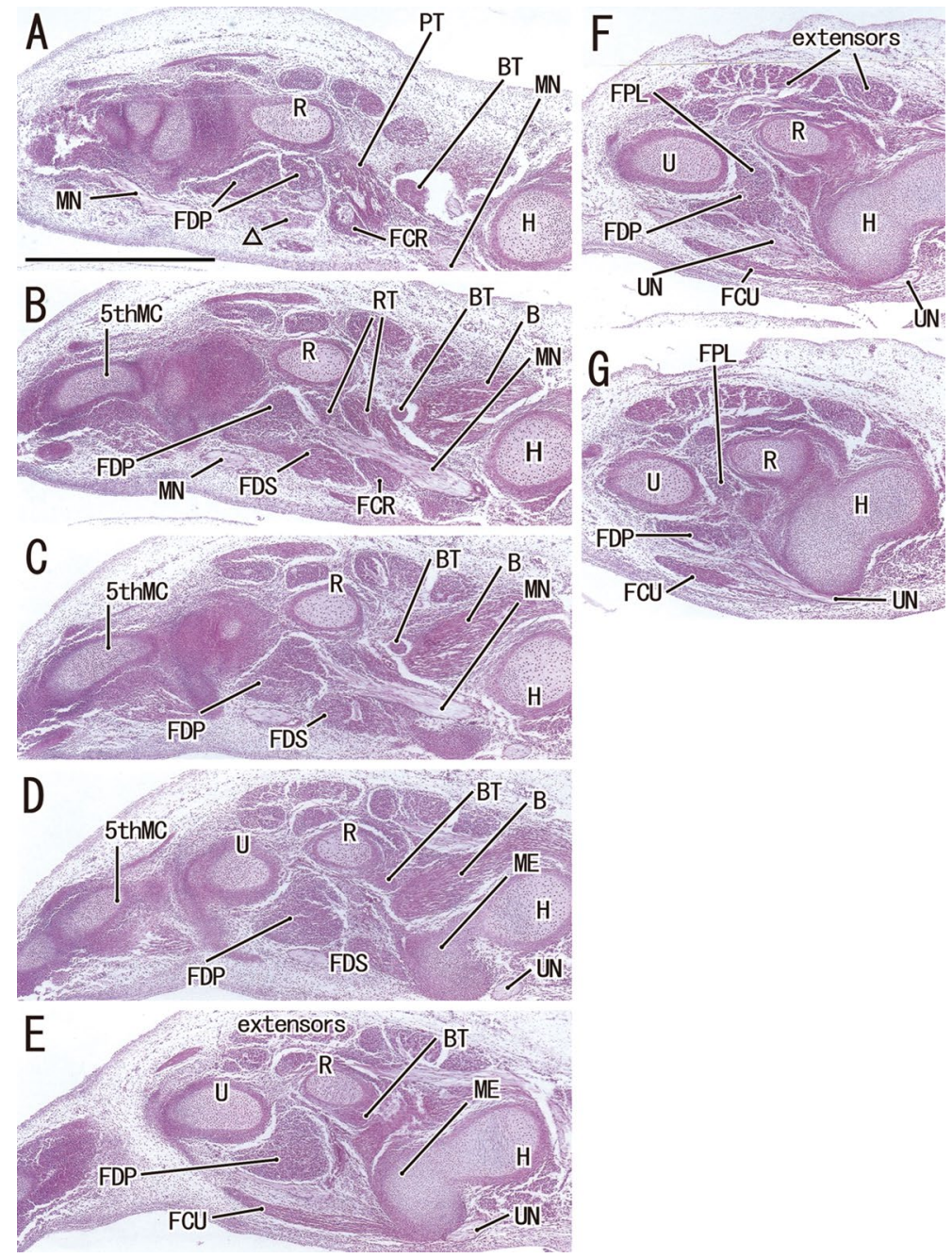

a candidate intermediate tendon (Fig. 8B), although the latter soon became difficult to distinguish from the usual intramuscular tendons in the FDS. Developing intramuscular tendons in the FDP extending into the FDS also prevented identification of the candidate intermediate tendon. However, in adults, communicating fascicles might not be reported between the FDS and FDP. Later, possibly after birth, a small muscle slip from the proximal anlage is covered by large muscle slips derived from the distal anlage (Fig. 8C). Thus, the distal anlage appeared to migrate proximally from the hand and wrist to the forearm near the elbow. At 6-7 weeks, however, these anlagen were not composed of undifferentiated myogenic cells but of primary myotubes. Because differentiated muscle fibres seem unable to migrate, drastic elongation of the insertion tendons of flexors was likely to compensate for the greater length of the radius and ulna, resulting in apparent proximal migration. The candidate intermediate tendon at 6-7 weeks may lengthen slightly.
Figure 5. A-G. Muscle anlagen in the elbow: oblique longitudinal sections from a specimen of crown rump length $25 \mathrm{~mm}$ (gestational age 7 weeks). The specimen shown here is identical to that in Figure 4. Panel $A$ is $0.1 \mathrm{~mm}$ to the ulnar and proximal side of Panel $F$ in Figure 4. A, G. The most distal and proximal sides of the figure, respectively. Intervals between panels are $0.2 \mathrm{~mm}$. The proximal anlage of the flexor digitorum superficialis (FDS) is evident (panels $B$ and $C$ ) on the proximal side of a candidate intermediate tendon of the FDS muscle (triangle in panel $\mathrm{A}$ ) and is attached to the medial epicondyle of the humerus (panel D). The median nerve ( $\mathrm{MN}$ in panel $\mathrm{B}$ ), as well as the ulnar nerve (UN in panel F), supply the flexor digitorum profundus muscle (FDP). All panels were prepared at the same magnification (scale bar in panel $A$, $1 \mathrm{~mm}$ ); $\mathrm{B}$ - brachialis muscle; $\mathrm{BT}$ - biceps tendon; FCU — flexor carpi ulnaris muscle; FDP flexor digitorum profundus muscle; $\mathrm{H}$ - humerus; $\mathrm{MC}$ - metacarpal bone; ME - medical epicondyle; $\mathrm{R}$ - radius; $\mathrm{U}$ - ulna.
This mechanism, however, cannot explain how the proximal anlage-derived muscle slip was covered by the distal anlage-derived major parts. Although the FDS has a double-layered configuration (see the 'Introduction'), further investigation was necessary to show the process of piling muscle slips. The macroscopic anatomy of the adult FDS indicates that the intermediate tendon should exist as a muscle slip for the second finger. However, our limited information on embryonic topographical relationships indicated that the distal anlage (i.e., the major parts of the FDS) was likely to develop in the "ulnar half" of the wrist and forearm, not in a limited tendon to the second finger.

The topographical anatomy of the muscles at and near the elbow joint apparently changes in late-stage foetuses and even after birth. The FDP was found to reach the elbow joint capsule, but the oblique cord was absent, suggesting that, possibly after birth, the proximal part of the foetal FDP had been replaced by 


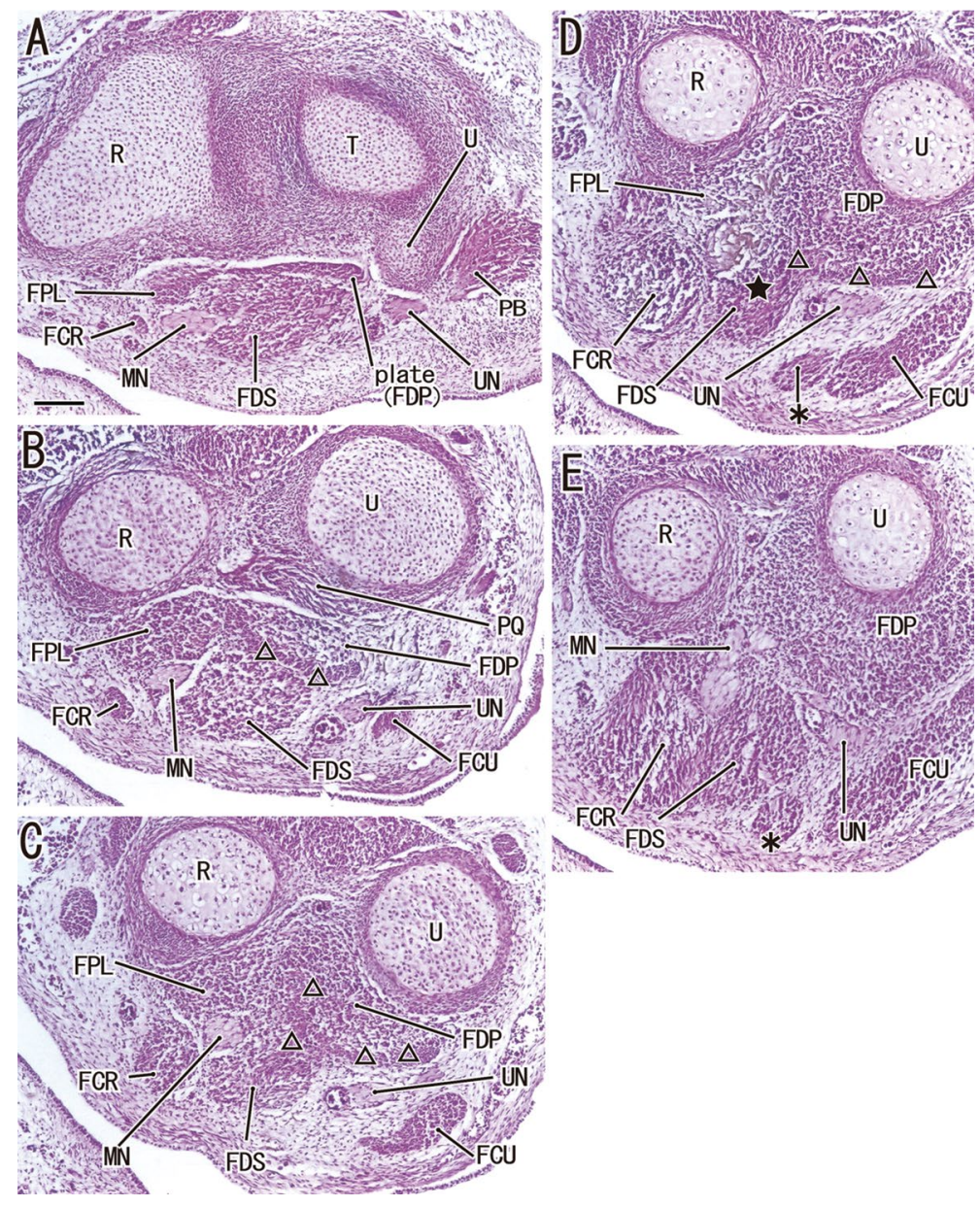

Figure 6. A-E. Cross sectional morphology of muscle anlagen at and near the wrist in a specimen of crown rump length $23 \mathrm{~mm}$ (gestational age 6 weeks). A, E. The most distal and proximal sides of the figure, respectively. Intervals between panels are $0.3 \mathrm{~mm}(A-B), 0.1 \mathrm{~mm}(B-C, C-D)$ and $0.2 \mathrm{~mm}(D-E)$. Panel A shows that, in contrast to the muscular mass of the flexor digitorum superficialis muscle (FDS), the flexor digitorum profundus muscle (FDP) is present as a tendinous plate (plate). On the proximal side, the deep muscle contains intramuscular tendon-like structures (triangles in panels $B-D)$, with parts of these structures extending into the FDS muscle. In panel D, almost the entire superficial muscles became tendinous (star; a candidate intermediate tendon). On the proximal side of a candidate intermediate tendon (panel $\mathrm{E}$ ), the FDS muscle attaches to the flexor carpi radialis muscle (FCR). The asterisks in panels $D$ and $E$ indicate an accessory bundle of the flexor carpi ulnaris muscle. The palmaris longus muscle is not found in this specimen. All panels were prepared at the same magnification (scale bar in panel $A, 0.1 \mathrm{~mm}$ ). FCU - flexor carpi ulnaris muscle; FPL — flexor pollicis longus muscle; $\mathrm{MN}$ - median nerve; $\mathrm{PB}$ - parmalis brevis; $\mathrm{PO}$ - pronator quadratus muscle; $\mathrm{R}$ - radius; $\mathrm{T}$ - triquetrum; $\mathrm{U}$ - ulna; UN — ulnar nerve. fibrous tissue to form the chord [8]. The consistent absence of the head in foetuses examined suggests that the aponeurotic origin of the ulnar head of the pronator teres may originate from the degenerated proximal part of the FDF because of the consistent absence of the head in foetuses examined [18]. This absence was also observed in this study. Similarly, we did not find that the FDS originated from the radius, suggesting the attachment may have occurred after changes in the topographical anatomy of muscles located near the elbow. The lumbricalis muscles are thought to originate from the FDS anlage [20], but develop later in accordance with a change from a single tendinous plate to five deep tendons in the hand [2].

\section{CONCLUSIONS}

Assessment of the foetal FDS failed to show development of the 3-dimensional muscle slip configuration or proximal migration in exchange for elongation of tendons. The FDS as a digastric muscle seemed to be "masked" not only in the surface view but by its complicated development processes. These findings indicate that the FDS was not simply an example of an embryonic, temporal digastric muscle, similar to the tensor tympani and tensor veli palatini muscles. Rather than the FDS, we expects a masked digastric muscle in the embryonic soleus because, in its deep or anterior aspect, the adult soleus is always accompanied by a feather-like, additional muscle belly with a short tendon merging with the Achilles tendon. In contrast to the major part of the soleus, which is innervated by thick, posterior or proximal branches, the feather-like belly is supplied by the thin, anterior or distal branch. Moreover, the latter nerve also supplied a rare variation, an accessory soleus with a tendon independent of the Achilles tendon $[16,17]$. Our preliminary observations showed that a pair of proximal anlagen near the knee and a distal anlage at the foot planta formed two layers. Thus, the superficial and deep digastric muscles were likely to present in the foot flexors. Finally, the plantaris muscle also contributed to the hypothetical double-layered digastric muscles, as the plantaris muscle corresponded to a long flexor of the fingers observed in marsupials [9]. 

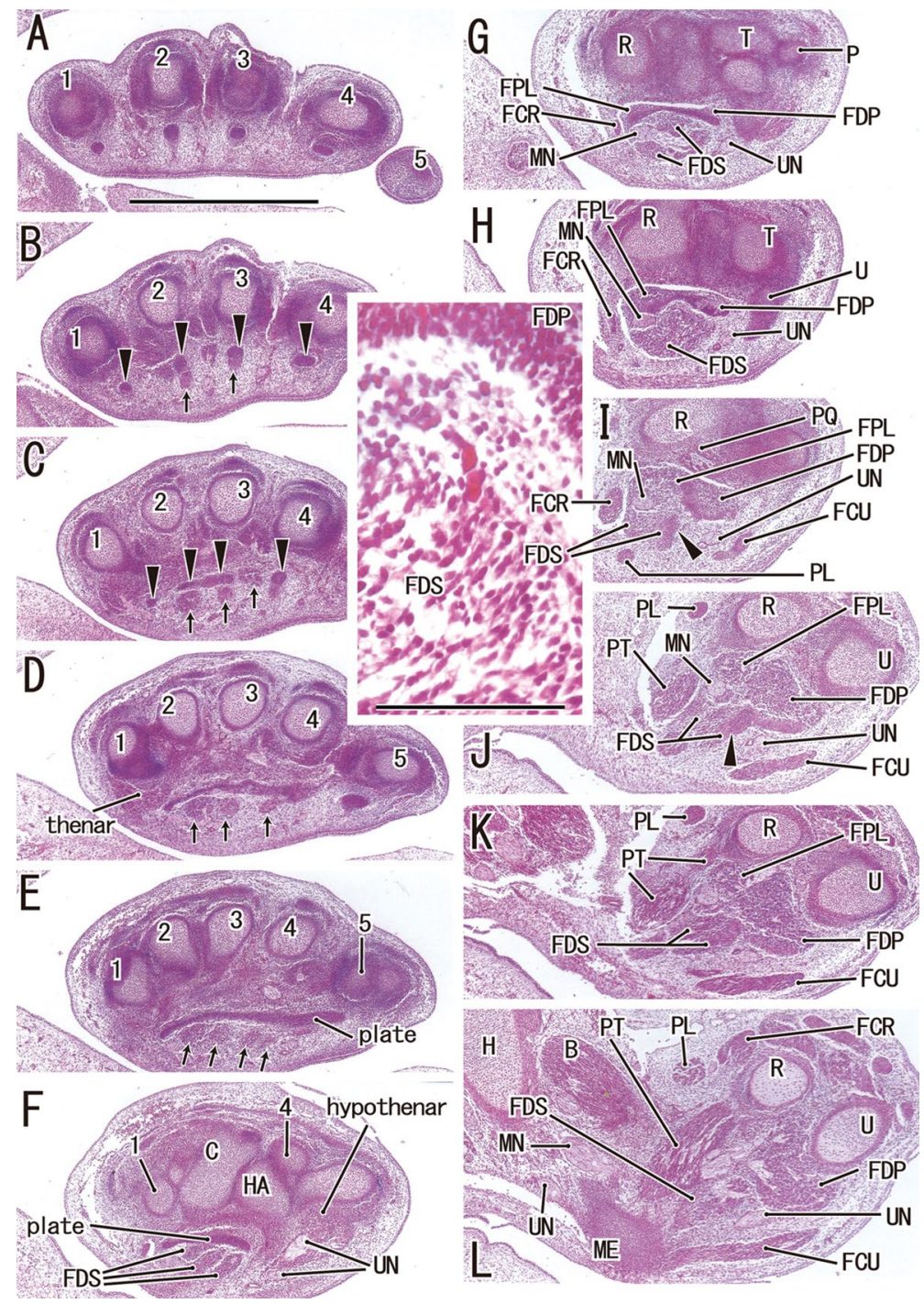

Figure 7. A-L. Cross sectional morphology of the finger, hand and forearm in a specimen of crown rump length $27 \mathrm{~mm}$ (gestational age 7 weeks).

A, L. The most distal and proximal sides of the figure, respectively. Intervals between panels are $0.1 \mathrm{~mm}$ (J-K), $0.2 \mathrm{~mm}$ (A-B, C-D, D-E, E-F, G-H, H-I. I-J, $\mathrm{K}-\mathrm{L})$, and $0.3 \mathrm{~mm}(\mathrm{~B}-\mathrm{C}, \mathrm{F}-\mathrm{G})$. Panels $\mathrm{A}-\mathrm{L}$ were prepared at the same magnification (scale bar in panel $A, 1 \mathrm{~mm}$ ), while an insert in the centre of the figure (scale bar, $0.1 \mathrm{~mm}$ ) is a higher magnification view of a square in panel $\mathrm{H}$. Five digital tendons of deep flexors (arrowheads) are well differentiated in panels $A$ and $B$, while tendons of the flexor digitorum superficialis muscle (FDS) are loose and poorly differentiated (arrows in panels B-E). The deep flexors make a tendinous plate (plate) in panels D-G. A thick tendinous bundle in the FDS (arrowheads in panels I and $\mathrm{J}$ ) is a candidate intermediate tendon. On the proximal side of a candidate intermediate tendon, the FDS was attached to the pronator teres muscle (PT). In these flexors, the primary myotubes appear to be differentiated (insert, centre). The palmaris longus muscle (PL) is seen on the radial side of the pronator; $\mathrm{B}$ - brachialis muscle; $\mathrm{C}$ - capitate; FCR — flexor carpi radialis muscle; $\mathrm{H}$ - humerus; $\mathrm{HA}$ - hamate; $\mathrm{ME}$ - medical epicondyle; MN — median nerve; $\mathrm{P}$ - pisiform; $\mathrm{PO}$ - pronator quadratus muscle; $\mathrm{R}$ - radius; $\mathrm{T}$ - triquetrum; $\mathrm{U}$ - ulna; $\mathrm{UN}$ ulnar nerve.

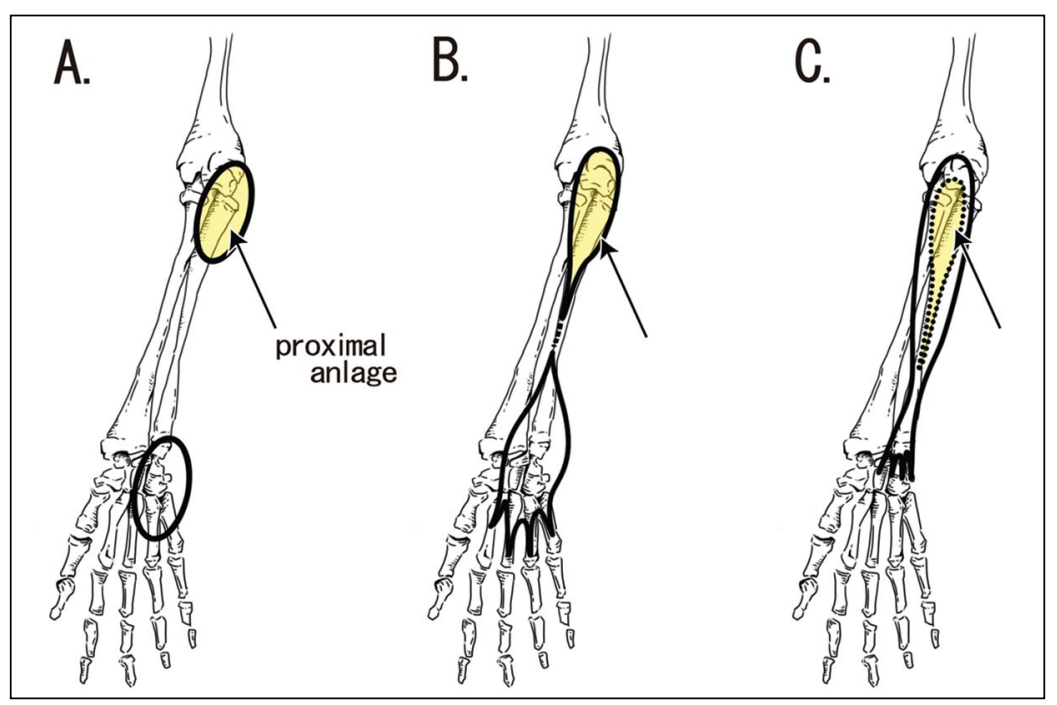

Figure 8. Diagram showing the hypothetical development of the flexor digitorum superficialis muscle. The distal anlage appears at the hand and wrist, while the proximal anlage appears at the elbow (A). At 6-7 weeks, these anlagen are connected by an intermediate tendon (B), but the latter soon becomes difficult to distinguish from usual intramuscular tendons. In adults (C), a small muscle slip from the proximal anlage (dotted line) is covered by large muscle slips derived from the distal anlage. 


\section{Acknowledgements}

This work was supported by the National Natural Science Foundation of China (81460471).

\section{REFERENCES}

1. Abe $M$, Murakami G, Noguchi $M$, et al. Variations in the tensor veli palatini muscle with special reference to its origin and insertion. Cleft Palate Craniofac J. 2004; 41(5): 474-484, doi: 10.1597/02-049.1, indexed in Pubmed: 15352865.

2. Cho KH, Kim JH, Ha YS, et al. Development of the deep flexor tendons and lumbricalis muscle in the hand and foot: a histological study using human mid-term foetuses. Folia Morphol (Warsz). 2012; 71(3): 154-163, indexed in Pubmed: 22936550.

3. Dylevský I. Tendons of the $m$. flexor digitorum superficialis et profundus in the ontogenesis of the human hand. Folia Morphol (Praha). 1968; 16(2): 124-130, indexed in Pubmed: 5649116.

4. Frohse $M$, Fränkel M. Die Muskeln des menschlichen Arms. In: Bardeleben, K. (ed), Handbuch der Anatomie des Menschen. Band II, Abt II, Teil II, Gustav Fischer, Jena. 1908: 127-133.

5. Gasc JP. Axial musculature. In Biology of the Reptilia. Gans C, Parsons TS (eds.). Academic press, London. 1981; 11: 355-435.

6. Gräfenberg E. Die Entwicklung der Knochen, Muskeln und Nerven der Hand und der für die Bewegungen der Hand bestimmten Muskeln des Unterarms. Beiträge und Referate zur Anatomie und Entwickelungsgeschichte. 1905; 30(1): 5-154, doi: 10.1007/bf02300781.

7. Huijing PA. Muscle as a collagen fiber reinforced composite: a review of force transmission in muscle and whole limb. J Biomech. 1999; 32(4): 329-345, indexed in Pubmed: 10213024.

8. Jin ZW, Jin $Y$, Yamamoto $M$, et al. Oblique cord (chorda obliqua) of the forearm and muscle-associated fibrous tissues at and around the elbow joint: a study of human foetal specimens. Folia Morphol. 2016; 75(4): 493-502, doi: 10.5603/FM.a2016.0019, indexed in Pubmed: 27830875.
9. Jin $\mathrm{ZW}$, Shibata $\mathrm{S}$, Abe $\mathrm{H}$, et al. A new insight into the fabella at knee: the foetal development and evolution. Folia Morphol. 2017; 76(1): 87-93, doi: 10.5603/ FM.a2016.0048, indexed in Pubmed: 27665955.

10. Katori Y, Hyun Kim Ji, Rodríguez-Vázquez JF, et al. Early fetal development of the intermediate tendon of the human digastricus and omohyoideus muscles: a critical difference in histogenesis. Clin Anat. 2011; 24(7): 843-852, doi: 10.1002/ca.21182, indexed in Pubmed: 21538565.

11. Lewis W. The development of the arm in man. Am J Anat. 1902; 1(2): 145-183, doi: 10.1002/aja.1000010204.

12. McMurrich J. The phylogeny of the forearm flexors. Am J Anat. 1903; 2(2): 177-209, doi: 10.1002/aja.1000020203.

13. Murakami G, Akita K, Sato T. Arrangement and innervation of the iliocostalis and longissimus muscles of the brown caiman (Caiman crocodilus fuscus: Alligatoridae, Crocodilia). Am J Anat. 1991; 192(3): 241-256, doi: 10.1002/ aja.1001920304, indexed in Pubmed: 1759688.

14. Ohtani O. Structure of the flexor digitorum superficialis. Okajimas Folia Anat Jpn. 1979; 56(5): 277-288, indexed in Pubmed: 547208.

15. Rodríguez-Vázquez JF, Sakiyama K, Abe H, et al. Fetal tendinous connection between the tensor tympani and tensor veli palatini muscles: a single digastric muscle acting for morphogenesis of the cranial base. Anat Rec (Hoboken). 2016; 299(4): 474-483, doi: 10.1002/ar.23310, indexed in Pubmed: 26744237.

16. Sekiya S, Kumaki K, Yamada TK, et al. Nerve supply to the accessory soleus muscle. Acta Anat (Basel). 1994; 149(2): 121-127, indexed in Pubmed: 8036872.

17. Sekiya S. Muscle architecture and intramuscular distribution of nerves in the human soleus muscle. Acta Anat (Basel). 1991; 140(3): 213-223, indexed in Pubmed: 1867062.

18. Tomita N, Yamamoto M, Sato M, et al. Topographical anatomy of the pronator teres muscle and medianus nerve: a study using histological sections of human fetuses. Okajimas Folia Anat Jpn. 2017; 94: 50-55.

19. Trotter JA. Functional morphology of force transmission in skeletal muscle. A brief review. Acta Anat (Basel). 1993; 146(4): 205-222, indexed in Pubmed: 8317197.

20. Yamada TK. [Re-evaluation of the flexor digitorum superficialis]. Kaibogaku Zasshi. 1986; 61 (4): 283-298, indexed in Pubmed: 3788478. 